An Upper Bound for the Linearized Map of an Inverse Problem for the Wave Equation

Gang Bao and

William W. Symes

February, 1991

TR91-2 



\title{
AN UPPER BOUND FOR THE LINEARIZED MAP OF AN INVERSE PROBLEM FOR THE WAVE EQUATION $^{1}$
}

\author{
Gang Bao and William W. Symes \\ Department of Mathematical Sciences \\ Rice University \\ Houston, Texas 77251-1892
}

\section{Introduction}

A simplified model which governs many physical processes such as seismic and acoustic wave propagation is the following linear acoustic wave equation:

$$
\left(\frac{1}{c^{2}} \frac{\partial^{2}}{\partial t^{2}}-\Delta-\nabla \sigma \cdot \nabla\right) u=f,
$$

where $\sigma=\sigma(x)$ is the logarithm of the density, $c=c(x)$ is the sound speed of the medium, and $f=f(x, t)$ is the source term which introduces the energy to the problem. If $\sigma, c$ and $f$ are given along with appropriate side conditions, the forward (or direct) problem is to determine $u=u(x, t)$, the excess pressure. For appropriate choices of $\sigma, c$, and $f, u$ is determined uniquely by standard linear hyperbolic theory of partial differential equations (p.d.e.). Thus the problem stated above defines a map from the coefficients to the solution of the wave equation. In this paper, we study an aspect of the regularity of this map, or rather its composition with the trace on a time-like hypersurface.

Throughout this work we shall restrict ourselves to the special case of constant velocity $c$, though we believe that the ideas in this work may be extended to cover some more general cases.

To fix ideas, write $x \in \mathbb{R}^{n}$ as $\left(x^{\prime}, x_{n}\right)$, where $x^{\prime} \in \mathbb{R}^{n-1}, x_{n} \in \mathbb{R}$. We assume that the problem is set in the whole space $\mathbb{I R}^{n}$ and $u=0$ in the past $(t<0)$. Take $f(x, t)=\delta(x, t)$ as an ideal point source. Thus $u$ is the retarded fundamental solution:

$$
\begin{aligned}
\square u & -\nabla \sigma \cdot \nabla u=\delta(x, t), \quad(x, t) \in \mathbb{R}^{n} \times \mathbb{R} \\
u & =0, t<0,
\end{aligned}
$$

where $\square$ is defined to be $\partial_{t}^{2}-\Delta$, and $\Delta$ is the Laplacian.

\footnotetext{
${ }^{1}$ This work was partially supported by the National Science Foundation under grant DMS 86-03614 and DMS 89-05878, by the Office of Naval Research under contracts N00014-K-85-0725 and N00014-J-89-1115, by AFOSR 89-0363, and by the Geophysical Parallel Computation Project (State of Texas).
} 
Define the forward map $F$ as:

$$
F:\left.\sigma \rightarrow(\phi u)\right|_{x_{n}=0}
$$

where $\phi \in C_{0}^{\infty}\left(\mathbb{R}^{n+1}\right)$ is supported inside the conoid $\{t>|x|\}$ and near $\left\{x_{n}=0\right\}$.

Because $F$ is nonlinear, one wants to work with the formal linearization (or formal derivative) $D F$, with respect to the reference state $\left(\sigma_{0}, u_{0}\right)$, defined by first order perturbation theory (Born-approximation).

$$
\begin{aligned}
\square \delta u & -\nabla \sigma_{0} \cdot \nabla \delta u=\nabla \delta \sigma \cdot \nabla u_{0} \\
\delta u & =0, t<0 .
\end{aligned}
$$

The formal derivative $D F\left(\sigma_{0}\right)$ is given by

$$
D F\left(\sigma_{0}\right) \delta \sigma=\left.(\phi \delta u)\right|_{x_{n}=0}
$$

It is our main goal in this work to determine appropriate spaces of the domain and range of $F$ for which

$$
\text { the formal derivative } D F \text { is bounded. }
$$

Throughout this paper, we shall always assume that

$$
\text { (A) } \operatorname{supp}(\delta \sigma) \subset\left\{x_{n}>\epsilon\right\} \text {, }
$$

for $\epsilon>0$ small. In some applications, this assumption is realistic, as the density can be measured directly, near the location of receivers (i.e. $x_{n}=0$ ). Also the domain of dependence properties of the wave equation implies that $\phi u$ and $\phi \delta u$ depend on $\sigma$ and $\delta \sigma$ only in a bounded subset. Thus we shall throughout assume that $\sigma$ and $\delta \sigma$ have fixed, bounded support.

Let $\Omega \subset \mathbb{R}^{k}$ be open and bounded, $\gamma \subset T^{*}(\Omega)$. A constant $C$ is said to depend on the $H^{s} \cap H_{m \ell}^{r}(\gamma)$-norm of $w \in C_{0}^{\infty}\left(\mathbb{R}^{k}\right)$ if for any conic neighborhood $\Gamma$ of $\gamma$ there exists a $\psi$.d.o. $Q$ of order zero with $E S(Q) \subseteq \Gamma$ and $q=1$ on $\gamma \cap\{(x, \xi):|\xi|>1\}$ such that $C$ can be bounded in terms of $\|w\|_{s, \Omega}+\|Q w\|_{r, \Omega}$.

The following is the statement of our main result which will be proved in the sections which follow.

Theorem 1.1 Assume either that

(i) $n \geq 3, s>\max \{3+n / 2, l+n-1\}$, and $\sigma_{0} \in H^{s}\left(\mathbb{R}^{n}\right)$ or

(ii) $n=2, s>\max \{4, l+1\}$, and $\sigma_{0} \in H^{s} \cap H_{m \ell}^{l+3 / 2}\left(\left\{(x, \xi): \xi_{n}=0\right\}\right)$.

Then under the assumption (A), the following estimate holds

$$
\left\|D F\left(\sigma_{0}\right) \delta \sigma\right\|_{l} \leq C\|\delta \sigma\|_{l+\frac{n-1}{2}}
$$

where the constant $C$ depends on the $H^{s}$-norm $(n \geq 3)$ or the $H^{s} \cap H_{m \ell}^{l+3 / 2}\left(\left\{(x, \xi): \xi_{n}=0\right\}\right)$ norm of $\sigma_{0}(n=2)$ but is independent of $\delta \sigma$. 
The study of the forward map is motivated by the inverse problem which arises in reflection seismology, oil exploration, ground-penetrating radar, etc. Mathematically, the inverse problem is to determine the coefficient $\sigma$ by knowing additional boundary value conditions of $u$. Since the inverse problem is just to invert the functional relation $F$, we are naturally interested in all the properties of this forward map.

To understand the problem, let us look at a simple exploration seismology experiment: Near the surface of the earth, a seismic source is fired at some point (point source). The seismic waves propagate into the earth. Since the earth's structure varies (as do its physical properties) part of the energy of the wave will be reflected back to the surface and can be measured. The inverse problem is to deduce the interior properties of the earth from the recorded data.

A simple model of this reflection seismic inverse problem in this context is: given data $F_{\text {data }}\left(x^{\prime}, t\right)$, find a coefficient $\sigma(x)$ so that

$$
F(\sigma)=F_{\text {data }}
$$

or perhaps minimizing the error $\left(F_{\text {data }}-F(\sigma)\right)$ in some norm.

Numerical solution of this problem by means of Newton's method and its relatives requires a choice of Banach space structure in the space of models $\sigma$ and in the space of data $F(\sigma)$ (see e.g. Kantorovich and Akilov [15]), in such a way that $F$ is singular. The simplest regularity property of $F$ is boundedness of $D F$, which is discussed in this paper. We believe that similar arguments will establish smoothness of $F$ and allow investigation of coercive properties of $D F$, as is required by the theory of optimization.

The most efficient relatives of Newton's method, the quasi-Newton, conjugate gradient, and variable metric methods, all require a Hilbert space structure on the space and models. This fact accounts for our reliance on the $L^{2}$-based Sobolev spaces in this work.

When the spatial dimension is one or $c$ and $\sigma$ depend only on $x_{n}$ (layered problem) there is a large literature available. For a similar problem in which the medium was assumed to be excited by an impulsive load on the surface $\left\{x_{n}=0\right\}$ instead of point sources, the properties of the forward map have been studied fairly satisfactorily by Symes and others (see Symes [25] for references). It was shown by Symes that, for the constant wave speed case, the forward map defines a $C^{1}$-diffeomorphism between open sets in certain Hilbert spaces by applying the method of geometrical optics together with energy estimates.

When the spatial dimension $n>1$ and $c, \sigma$ depend on all space variables (nonlayered problem), very little is known in mathematics. Symes [23, 24], Sacks and Symes [21], Rakesh [18], and Sun [22] have some partial results. The difficulties are essentially due to the ill-posed nature of the timelike hyperbolic Cauchy problem and the presence of nonsmooth coefficients. For the one dimensional wave equation, both coordinate directions are spacelike, which indicates that the problem is hyperbolic with respect to both directions. Apparently, this is not the case when the spatial dimension is larger than one.

Rakesh in [18] looked at a related linearized velocity inversion problem with constant density and point sources. Assuming smooth background velocity, he obtained some results on both upper and lower bounds for the linearized forward map. The essential observation in Rakesh's work is that $D F$ is a Fourier integral operator (see also Beylkin [7]). Unfortunately, 
the calculus of Fourier integral operators employed in Rakesh's work is not applicable to the nonsmooth reference velocity case since the linearized forward map is a Fourier integral operator only when the reference velocity is smooth.

In [23], Symes gave a pair of examples, based on the geometric optics construction, which show that both $D F(1)$ and $D F(1)^{-1}$ are unbounded for a slightly different problem. As the examples show, within the Sobolev scales no strengthening or weakening of topologies of the domain and range can make both $D F$ and $D F^{-1}$ bounded. This fact also implies a strategy of regularization: Change the topology in the domain so that $D F$ becomes bounded, then ask for optimal regularization of $D F^{-1}$ in the sense of best possible lower bound estimate for $D F$. In both examples of Symes, the unboundedness was caused by rapid oscillation of $\sigma$ in the $x^{\prime}$-direction or the tangential directions, hence the problem is actually "partially well-posed", i.e., only more smoothness of the coefficients in tangential directions (essentially grazing ray directions) will be required to cure the difficulty. This might be the main reason the anisotropic Sobolev spaces $H^{m, s}\left(\mathbb{R}^{n}\right)$ or Hörmander spaces, were introduced in [21] and [22].

In Theorem 4.1 of [21] Sacks and Symes showed by using the method of sideways energy estimates that for a linearized density determination problem with constant velocity and plane wave sources, $D F$ is bounded from $H^{1,1}$ to $H^{1}$, provided the reference coefficient is in $H^{1, s}$ for some $s>n+2$. They also proved the injectivity of $D F$. Our techniques and results are quite different from theirs. We intend to assure the optimal regularity of the timelike trace under different hypotheses, which are in some ways weaker.

There remains an extremely important issue to be addressed, namely,

What is an appropriate space for the domain of DF?

In 1983, Symes suggested that microlocal restrictions on the coefficients might regularize the inverse problem (see [24]). In some sense, this was confirmed by Bao and Symes [2] where we were able to prove a trace theorem for the solutions of general linear p.d.e. with smooth coefficients. Roughly speaking, our theorem asserts that the solution will belong to $H^{s}$ along a codimension one hypersurface if it belongs to $H^{s}$ in a neighborhood of the hypersurface and to $H^{s+1}$ microlocally in those directions where the p.d.e. is not microlocally strictly hyperbolic. Note that we gained back the half derivative from the standard trace theorem. In a recent paper [3], we proved a similar time like trace regularity result for a second order hyperbolic equation with nonsmooth coefficients. It is obvious that the presence of nonsmooth coefficients will introduce new singularities to the solutions so that only limited initial regularity can be propagated. A crucial step in [3] was to develop an extended Beals-Reed theorem (Theorem 1 in [6]) on propagation of singularities.

The main result of this paper is a boundedness theorem for the linearized forward map $D F\left(\sigma_{0}\right)$ for the (sufficiently regular) nonsmooth $\sigma_{0}$. The main ingredients of our proof are the method of energy estimates, a microlocal regularity study of the fundamental solution, results on propagation of singularities, several trace regularity results, and a useful duality technique.

It is known that in their applications to nonlinear wave equations, most of the results based on Rauch's lemma (or the method of Fourier analysis) are limited to relatively weak singularities. This work exhibits that to some extent, strong singularities appearing in the 
linear wave equation (e.g. the fundamental solution) can also be tackled by this Fourier analysis method with the help of a duality argument and the progressing wave expansion. The relation between the coefficients and solution with strong singularities remains to be fully understood, especially when the coefficients are less regular.

The plan of this paper is as follows. In Section 2, a regularity theorem for the solution of the model problem is established. To serve this purpose, a dual problem is introduced. A crucial step is the derivation of an explicit estimate from Hömander's result on propagation of singularities. Section 3 is devoted to the proof of our main theorem. The main ingredients of our proof are: microlocal regularity analysis of the solutions for the transport equations; a microlocal version of the classical trace theorem; and regularity study of the dual problem.

Notation. Throughout this paper, the reader is assumed to be familiar with the basic

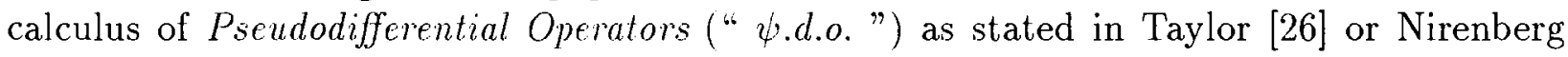
[17]. A classical 4.d.o. $P$ of order $m$ is denoted as $P \in O P S^{m}$ with its symbol $p \in S^{m}$. $E S(P)$ stands for the esscntial support of operator $P$. WF $(u)$ denotes the wave front set of a distribution $u . H^{s}$ is the standard $L^{2}$-type Sobolev space and $H_{\text {loc }}^{s}$ means a local Sobolev space. $\langle\xi\rangle$ means $\left(1+|\xi|^{2}\right)^{1 / 2}$. For a nice discussion on microlocal Sobolev spaces $H^{s} \cap H_{m \ell}^{r}\left(x_{0}, \xi_{0}\right)$, we refer the reader to Beals [5], see also Rauch [19]. For simplicity, $C$ serves as a generalized positive constant the precise value of which is not needed.

Warning. When the reference density $\sigma_{0}$ is smooth, most of the regularity results for the forward map in this work will follow more easily from the calculus of Fourier Integral Operators. For a standard text on F.I. O. we refer to Duistermaat [11] or Hörmander [13]. However, this technique fails with the appearance of the nonsmooth reference density, an assumption important in this work.

\section{Regularity of the Fundamental Solution}

Since the excess pressure $u$ in the model equation is in fact the fundamental solution, in order to study the regularity of the forward map, the regularity of the fundamental solution must be understood. It is evident that the real obstacle here is the singular right-hand side so that none of the propagation of singularity results could be applied directly. A natural way to cure this difficulty is by employing the Hadamard theory of progressing wave expansion. We refer the reader to Courant and Hilbert [10] or Friedlander [12] for a detail study on the method of progressing wave expansions. According to Hadamard's construction, the fundamental solution may be represented as a sum of the principal part and remainder. One can then study the remainder by the Beals-Reed type propagation of singularity theorem. However, a great drawback of this idea is that additional regularity is needed to regularize the remainder term. In this section, we develop a new approach based on the method of microlocal analysis. A dual problem is introduced so that the regularity study of the fundamental solution may be transformed into regularity study of the dual problem which has a smooth right hand side. In this process, a crucial step is to derive an estimate out of Hörmander's theorem on propagation of singularities. As one might expect, with the presence of nonsmooth coefficients, the Rauch-type results and some commutator results will be demanded. 


\subsection{Statement of result, preliminaries}

Consider a problem obtained by integrating the model problem in the time variable,

$$
\begin{aligned}
& \left(\square-\nabla \sigma_{0} \cdot \nabla\right) v_{0}=\delta^{-\frac{n-1}{2}}(t) \delta(x), \quad(x, t) \in \mathbb{R}^{n+1} \\
& v_{0}=0, \quad t<0 .
\end{aligned}
$$

The following is a regularity theorem for the fundamental solution.

Theorem 2.1 Suppose that $1+n / 2<s$ and $\sigma_{0} \in H^{s}\left(\mathbb{R}^{n}\right)$. Then for $v_{0}$ solving the equation (2.1),$l<s-n / 2$

$$
v_{0} \in H_{l o c}^{l}(U)
$$

where $U=\left\{\mathbb{R}^{n} \times\left(0, T_{1}\right)\right\} \cap\{t>|x|\}\left(T_{1}>0\right)$. And for $\phi \in C_{0}^{\infty}(U)$, the following estimate holds

$$
\left\|\phi v_{0}\right\|_{l} \leq C
$$

where the constant $C$ depends on $\phi$ and $\left\|\sigma_{0}\right\|_{s}$.

In order to establish Theorem 2.1, we need the following results. The first was originally established by Bony [8] and was extended by Meyer [16]. See also Beals [4] for a different proof.

Proposition 2.1 Suppose that for some $\left(x_{0}, \xi_{0}\right) \in T^{*}\left(\mathbb{R}^{n}\right) \backslash 0, u \in H^{s} \cap H_{m \ell}^{r}\left(x_{0}, \xi_{0}\right), n / 2<$ $s \leq r \leq 2 s-n / 2$, and $g \in C^{\infty}$, then

$$
g(x, u) \in H^{s} \cap H_{m \ell}^{r}\left(x_{0}, \xi_{0}\right) .
$$

We also need a Gårding's type inequality concerning the microlocal ellipticity.

Lemma 2.1 Assume that $Q_{1} \in O P S^{m_{1}}, Q_{2} \in O P S^{m_{2}}$, with $m_{1}, m_{2} \in \mathbb{R}$. Furthermore assume that $Q_{2}$ is elliptic on $E S S^{\prime}\left(Q_{1}\right)$. Then for any $r \in \mathbb{R}, \Omega$ and $\Omega^{\prime}$ two open bounded sets of $\mathbb{R}^{n}$ with $\Omega \subset \subset \Omega^{\prime}$, and $u \in C_{0}^{\infty}(\Omega)$,

$$
\left\|Q_{1} u\right\|_{s, \Omega} \leq C\left\|Q_{2} u\right\|_{s+m_{1}-m_{2}, \Omega^{\prime}}+C\|u\|_{r, \Omega^{\prime}} .
$$

Proof. Let $\Omega_{1}$ and $\Omega_{2}$ be opell sets with $\Omega \subset \subset \Omega_{1} \subset \subset \Omega_{2} \subset \subset \Omega^{\prime}$. Construct a cut-off function $\phi \in C_{0}^{\infty}\left(\Omega^{\prime}\right), \phi=1$ on $\Omega_{1}$, and $\phi=0$ on $\Omega^{\prime} \backslash \Omega_{2}$.

The assumption $Q_{2}$ is elliptic on $E S\left(Q_{1}\right)$ implies that a $\psi$.d.o $R$, a parametrix of $Q_{2}$ on $E S\left(Q_{1}\right)$, may be found such that

$$
Q_{1} R Q_{2}=Q_{1}+K
$$

with $K$ a smoothing operator.

Having defined $\phi$, we can now rewrite

$$
Q_{1} R Q_{2} u=Q_{1} R \phi Q_{2} u+Q_{1} R(1-\phi) Q_{2} u \cdot v
$$


It follows that, for any $r$,

$$
\begin{aligned}
\left\|Q_{1} R Q_{2} u\right\|_{s, \Omega} & \leq\left\|Q_{1} R \phi Q_{2} u\right\|_{s, \Omega}+\left\|Q_{1} R(1-\phi) Q_{2} u\right\|_{s, \Omega} \\
& \leq C\left\|\phi Q_{2} u\right\|_{s+m_{1}-m_{2}, \mathbb{R}^{n}+C\left\|(1-\phi) Q_{2} u\right\|_{r, \Omega^{\prime}}} \\
& \leq C\left\|Q_{2} u\right\|_{s+m_{1}-m_{2}, \Omega^{\prime}}+C\|u\|_{r, \Omega^{\prime}}
\end{aligned}
$$

On the other hand, from (2.3), it is obvious that

$$
\left\|Q_{1} R Q_{2} u\right\|_{s, \Omega} \geq\left\|Q_{1} u\right\|_{s, \Omega}-\|K u\|_{s, \Omega}
$$

A combination of the above discussions will complete the proof.

Finally, the proof of Theorem 2.1 demands the use of the following two lemmas. Lemma 2.2 gives an estimate based on Nirenberg's proof [17] of Hörmander's theorem which describes the propagation of regularity along bicharacteristics. With nonsmooth coefficients, only a limited amount of regularity propagates. It indicates that an estimate may be derived near any bicharacteristic, hence near the characteristic variety of operator $\square=\partial_{t}^{2}-\Delta$. We then proceed in Lemma 2.3 to argue that in the elliptic region of the operator $\square$ an estimate may also be formed. With a concern about the nonsmooth $\sigma_{0}$, it should not be surprising that both results require a commutator argument.

Let $\Pi: T^{*}\left(\Omega_{0}\right) \rightarrow \Omega_{0}$ denote the projection of $T^{*}\left(\Omega_{0}\right)$ onto its base space.

Lemma 2.2 Assume that $s>n / 2, \sigma_{0} \in H^{s+1}\left(\mathbb{R}^{n}\right)$. Suppose that $\beta$ is a null bicharacteristic of $\square,\left(x_{0}, \xi_{0}\right) \in \beta, w$ is smooth in a neighborhood of $x_{0}$, and

$$
\square w-\nabla \sigma_{0} \cdot \nabla w \in L^{2}\left(\mathbb{R}^{n+1}\right)
$$

vanishes in a neighborhood of $\Pi \beta$. Then there exists a $\psi . d . o$. B of order zero (essentially supported near $\beta$ ) so that for any $\phi_{1} \in C_{0}^{\infty}\left(\mathbb{R}^{n+1}\right)$ and $k<s-n / 2+2$, there is $C>0$,

$$
\left\|\phi_{1} B w\right\|_{k} \leq C\left\|\square w-\nabla \sigma_{0} \cdot \nabla w\right\|_{0}
$$

Here $C$ depends on $\sigma_{0}, k, B$, and $\phi_{1}$, but not on $w$.

Proof. Let $\Omega$ be an open bounded set containing $\operatorname{supp}\left(\phi_{1}\right)$ and

$$
\square w-\nabla \sigma_{0} \cdot \nabla w=f \text {. }
$$

According to Nirenberg's construction, one can find a $\psi$.d.o. $B_{0}$ of order zero with

(1) $b_{0}$ supported in a small conic neighborhood of $\beta, B_{0}$ elliptic near $\beta$,

(2) $\prod \operatorname{supp}\left(b_{0}\right) \cap \operatorname{supp}(f)=\emptyset$, and

(3) $\left[\square, B_{0}\right] \in O P S^{\circ}$ 
Since $w$ solves (2.1), the method of energy estimates yields

$$
\|w\|_{1} \leq C\|f\|_{0}
$$

where $C$ is a constant depending on $\left\|\nabla \sigma_{0}\right\|_{s}$ for $\check{s}>n / 2$.

Observe that from (2.1),

$$
\square_{\mathrm{l}}^{\prime} B_{0} w=\left[\square, B_{0}\right] w+\left[B_{0}, \nabla \sigma_{0} \cdot \nabla\right] w+B_{0} f .
$$

Since $\Pi \operatorname{supp}\left(b_{0}\right) \cap \operatorname{supp}(f)=\emptyset$, we have

$$
B_{0} f=0
$$

Now energy estimates give

$$
\left\|B_{0} w\right\|_{2, \Omega} \leq C\left(\left\|\left[\square, B_{0}\right] w\right\|_{1, \Omega}+\left\|\left[B_{0}, \nabla \sigma_{0} \cdot \nabla\right] w\right\|_{\mathbf{I}, \Omega}\right) .
$$

Since $\left[\square, B_{0}\right]$ is of order 0 ,

$$
\left\|\left[\square, B_{0}\right] w\right\|_{1, \Omega} \leq C\|w\|_{1} \leq C\|f\|_{0}
$$

The third term in (2.4) may be estimated by applying the generalized commutator lemma, Lemma 2.4 in [3] and the corresponding estimate. In fact, let $1+n / 2<s_{0}$, we then have

$$
\left\|\left[B_{0}, \nabla \sigma_{0} \cdot \nabla w\right]\right\|_{1, \Omega} \leq C\|w\|_{1} \leq C\|f\|_{0}
$$

where $C$ depends on $\left\|\nabla \sigma_{0}\right\|_{s_{0}}$.

Thus

$$
\left\|B_{0} w\right\|_{2, \Omega} \leq C_{0}\|f\|_{0}
$$

with $C_{0}$ depending on $\left\|\nabla \sigma_{0}\right\|_{s_{0}}$.

Applying Nirenberg's construction once again, we can find a $\psi$.d.o. $B_{1}$ of order zero such that $E S\left(B_{1}\right) \subset E S\left(B_{0}\right)$ (strictly), $B_{1}$ also has properties $(1)$ and $(2)$ above; moreover $\left[\square, B_{1}\right] \in O P S^{-1}$ and $B_{0}$ is elliptic near $E S\left(B_{1}\right)$. From (2.1) and $B_{1} f=0$,

$$
\square_{1}^{\prime} B_{1} w=\left[\square, B_{1}\right] w+\left[B_{1}, \nabla \sigma_{0} \cdot \nabla\right] w .
$$

If we write down the energy estimates, after a simple $\psi$.d.o. cut-off on $B_{1}$, we will find

$$
\left\|B_{1} w\right\|_{3, \Omega}^{2} \leq C\|w\|_{1}^{2}+C\left\|A_{1}\left[B_{1}, \nabla \sigma_{0} \cdot \nabla\right] w\right\|_{2, \Omega}\left\|B_{1} w\right\|_{3, \Omega},
$$

where $A_{1} \in O P S^{0}, E S\left(B_{1}\right) \subset E S\left(A_{1}\right) \subset E S\left(B_{0}\right), B_{0}$ is elliptic on $\operatorname{ES}\left(A_{1}\right)$, and $a_{1}=1$ on $E S\left(B_{1}\right) \cap\{(x, \xi),|\xi| \geq 1\}$.

Now since $w \in H^{1} \cap H_{m c}^{2}\left(E S\left(B_{0}\right)\right)$, Lemma 2.4 in [3] again implies that $\left[B_{1}, \nabla \sigma_{0} \cdot \nabla\right] w \in$ $H^{1} \cap H_{m \ell}^{2}\left(E S\left(A_{1}\right)\right)$ and

$$
\left\|A_{1}\left[B_{1}, \nabla \sigma_{0} \cdot \nabla\right] w\right\|_{2, \Omega} \leq C\left(\|w\|_{1}+\left\|A_{1} w\right\|_{2, \Omega}\right) .
$$

Here $C$ depends on $\left\|\nabla \sigma_{0}\right\|_{s_{1}}$ for $2+n / 2 \leq s_{1}$. 
Because of our construction, $B_{0}$ is elliptic on $E S\left(A_{1}\right)$; therefore Gårding's type inequality Lemma 2.1 leads to, for any real $r$ and $\Omega \subset \subset \Omega_{1}$

$$
\left\|A_{1} w\right\|_{2, \Omega} \leq C\left\|B_{0} w\right\|_{2, \Omega_{1}}+C\|w\|_{r} \leq C\|f\|_{0}
$$

by $(2.5)$.

Therefore we have shown that

$$
\left\|B_{1} w\right\|_{3, \Omega} \leq C_{1}\|f\|_{0}
$$

where $C_{1}$ depends on $\left\|\nabla \sigma_{0}\right\|_{s_{1}}$.

We can continue this process by constructing a sequence of $\psi$.d.o. $B_{i}$ and $A_{i}(i=1, \cdots$ $\cdot, k-2)$, such that

- $B_{i}$ has properties (1), (2), and $\left[\square, B_{i}\right] \in O P S^{-i}$,

- $E S\left(B_{i-1}\right) \subset E S\left(A_{i-1}\right) \subset E S\left(B_{i}\right)$, and

- $B_{i}$ is elliptic on $E S\left(A_{i-1}\right), a_{i-1}=1$ on $E S\left(B_{i-1}\right) \cap\{(x, \xi),|\xi| \geq 1\}$,

- Also

$$
\left\|\left.B_{i} w^{\prime}\right|_{i+2, \Omega} \leq C_{i}\right\| f \|_{0},
$$

where $C_{i}$ depends on $\left\|\nabla \sigma_{0}\right\|_{s_{1}}$ for $i+n / 2<s_{i}$.

Eventually we conclude by choosing $B=B_{k-2}$ so that, for $k-2+n / 2<s$,

$$
\|B w\|_{k, \Omega} \leq C\|f\|_{0}
$$

with $C$ depending on $\left\|\nabla \sigma_{0}\right\|_{s}$.

The proof of Theorem 2.1 requires a slightly different form of Lemma 2.2.

Corollary 2.1 Assume that $s>n / 2, \sigma_{0} \in H^{s+1}\left(\mathbb{R}^{n}\right)$. Suppose that $\gamma$ is a set of null bicharacteristics of $\square,\left(x_{0}, \xi_{0}\right) \in \gamma, w$ is smooth in a neighborhood of $x_{0}$, and

$$
\square w-\nabla \sigma_{0} \cdot \nabla w \in L^{2}\left(\mathbb{R}^{n+1}\right)
$$

vanishes in a neighborhood of $\Pi \gamma$. Then there exists a $\psi$.d.o. $Q$ of order zero (essentially supported near $\gamma$ ) so that for any $\phi_{1} \in C_{0}^{\infty}\left(\mathbb{R}^{n+1}\right)$ and $k<s-n / 2+2$, there is $C>0$,

$$
\left\|\phi_{1} Q w\right\|_{k} \leq C\left\|\square w-\nabla \sigma_{0} \cdot \nabla w\right\|_{0} .
$$

Here $C$ depends on $\sigma_{0}, k, Q$, and $\phi_{1}$, but not on $w$.

Proof. For every null bicharacteristic of the set $\gamma$, Lemma 2.2 indicates that a $\psi$.d.o. $B$ of order zero may be found so that.

$$
\left\|\phi_{1} B w\right\|_{k} \leq C\left\|\square w-\nabla \sigma_{0} \cdot \nabla w\right\|_{0} .
$$

Now $Q$ may be constructed as $Q=\sum B$. Moreover, the local compactness of the unit sphere ensures that the summation is finite. 
Lemma 2.3 Assume that $s>n / 2, \sigma_{0} \in H^{s+1}\left(\mathbb{R}^{n}\right)$. Suppose that $P$ is a $\psi$.d.o. of order zero such that a conic neighborhood of its essential support is contained in the elliptic region of the wave operator $\square$. Assume also that

$$
\square w-\nabla \sigma_{0} \cdot \nabla w \in L^{2}\left(\mathbb{R}^{n+1}\right)
$$

vanishes in a neighborhood of $\Pi p$. Then for any $\phi_{1} \in C_{0}^{\infty}\left(\mathbb{R}^{n+1}\right)$ and $k<s-n / 2+2$, there is $C>0$ so that

$$
\left\|\phi_{1} P w\right\|_{k} \leq C\left\|\square w-\nabla \sigma_{0} \cdot \nabla w\right\|_{0} .
$$

Here the constant $C$ depends on $\sigma_{0}, k, P$, and $\phi_{1}$, but not on $w$.

Proof. The proof is based on the same type of bootstrap arguments as in the proof of last lemma.

Let

$$
\square w-\nabla \sigma_{0} \cdot \nabla w=f .
$$

Assume that $\Omega \supset \operatorname{supp}\left(\phi_{1}\right)$ be a bounded open set. From the support assumption on $p$, we see that $P f=0$. Hence, by applying $P$ to both sides of (2.6), we find

$$
\square P w=[\square, P] w+\left[P, \nabla \sigma_{0} \cdot \nabla\right] w+\nabla \sigma_{0} \cdot \nabla P w .
$$

Now since $\square$ is elliptic in a small conic neighborhood of $E S(P)$, there exists a $\psi$.d.o. $P_{0}$ of order zero, such that $E S(P) \subset E S\left(P_{0}\right), P_{0}$ is elliptic near $E S(P)$, and $\square$ is elliptic in a small conic neighborhood of $E S\left(P_{0}\right)$. From the ellipticity of $P_{0} \square$ on $E S(P)$, Lemma 2.1 gives, for any real number $r$ and $\Omega \subset \subset \Omega^{\prime}$.

$$
\|P w\|_{k, \Omega} \leq C^{\prime}\left\|P_{0} \square P w\right\|_{k-2, \Omega^{\prime}}+C\|w\|_{r}
$$

or from $(2.7)$

$$
\|P w\|_{k, \Omega} \leq C\left(\left\|P_{0}[\square, P] w\right\|_{k-2, \Omega^{\prime}}+\left\|P_{0}\left[P, \nabla \sigma_{0} \cdot \nabla\right] w\right\|_{k-2, \Omega^{\prime}}+\left\|P_{0} \nabla \sigma_{0} \cdot \nabla P w\right\|_{k-2, \Omega^{\prime}}\right) .
$$

Therefore an application of Lemma 2.4 and the generalized Rauch's lemma in [3] yields

$$
\begin{aligned}
\|P w\|_{k, \Omega} \leq & C_{1}\left\|P_{0} w\right\|_{k-1, \Omega^{\prime}}+C_{2}\left(\|w\|_{1}+\left\|P_{0} w\right\|_{k-2, \Omega^{\prime}}\right) \\
& +C_{3}\left(\|w\|_{1}+\left\|P_{0} w\right\|_{k-1, \Omega^{\prime}}\right) \\
\leq & C\|f\|_{0}+C\left\|P_{0} w\right\|_{k-1, \Omega^{\prime}} .
\end{aligned}
$$

Here constants $C_{2}$ and $C_{3}$ depend on $\left\|\nabla \sigma_{0}\right\|_{s}$ for $k-2+n / 2<s$.

Thus the bootstrap arguments on $P_{0}$ will accomplish the proof. 


\subsection{Proof of Theorem 2.1}

We study the regularity of $v_{0}$ through its dual problem. To simplify the arguments on the dual problem, we make use of the symmetric form of (2.1) by introducing $\rho(x)=e^{-\sigma_{0}}$. Then (2.1) becomes

$$
\begin{aligned}
\square_{1} v_{0} & =\left[\frac{1}{\rho} \partial_{t}^{2}-\nabla \cdot\left(\frac{1}{\rho} \nabla\right)\right] v_{0}=\frac{1}{\rho} \delta^{-\frac{n-1}{2}}(t) \delta(x) \\
v_{0} & =0 \quad t<0 .
\end{aligned}
$$

Now let us look at a dual problem to $(2.8)$,

$$
\begin{aligned}
\square_{1} w & =\left[\frac{1}{\rho} \partial_{t}^{2}-\nabla \cdot\left(\frac{1}{\rho} \nabla\right)\right] w=\Psi \\
w & =0 \quad t>>T_{1},
\end{aligned}
$$

where $\Psi \in C_{0}^{\infty}(\Omega)$ with $\Omega=\left\{\mathbb{I R}^{n} \times\left(0 . T_{1}\right)\right\} \cap\left\{t>|x|+\epsilon_{0}\right\}$, for $\epsilon_{0}>0$ small. Note that, this equation may be reformulated as

$$
\begin{aligned}
& \square_{1}^{\prime} w=\square w-\nabla \sigma_{0} \cdot \nabla w=e^{-\sigma_{0}} \Psi \\
& w=0 \quad t>>T_{1} .
\end{aligned}
$$

Thus if we can show that for any $\Psi \in C_{0}^{\infty}(\Omega)$

$$
\left|\left(\partial_{l}^{l} v_{0}, \Psi\right)\right| \leq C|| \Psi \|_{0}
$$

then it can be concluded that

$$
\left\|\partial_{t}^{l} v_{0}\right\|_{0, \Omega} \leq C
$$

From (2.8), integration by parts leads

$$
\begin{aligned}
\left|\left(\partial_{t}^{l} v_{0}, \Psi\right)\right| & =\left|\left(\square_{1} \partial_{t}^{l} v_{0}, w\right)\right| \\
& =\left|\left(\frac{1}{\rho} \delta(t) \delta(x), \partial_{t}^{l-(n-1) / 2} w\right)\right| \\
& \leq C\left|\left(\partial_{t}^{l-(n-1) / 2} w\right)(0,0)\right| .
\end{aligned}
$$

The trace theorem (see for example [26]) yields that

$$
\left|\left(\partial_{t}^{l} v_{0}, \Psi\right)\right| \leq C\left\|\phi_{1} w\right\|_{l+1}
$$

with $\phi_{1} \in C_{0}^{\infty}\left(\Omega_{1}\right), \Omega_{1}$ a small neighborhood of the origin and $\Omega_{1} \cap \operatorname{supp}(\Psi)=\emptyset$.

Construct two \&.d.o. $Q_{1}, Q_{2} \in O P S^{0}\left(\mathbb{R}^{n+1}\right)$, such that

- $Q_{1}+Q_{2}=R$; where $R$ is an elliptic $\psi$.d.o. of order zero in $\Omega_{1}$;

- $\Pi \operatorname{supp}\left(q_{i}\right) \cap \operatorname{supp}(\Psi)=\emptyset$, for $i=1,2$;

- $E S\left(Q_{2}\right)$ is a small conic neighborhood of set of null bicharacteristics of the wave operator $\square$ passing over $\Omega_{1}$; 
- $Q_{1}$ is microlocally smoothing on the null bicharacteristics passing over $\Omega_{1}$.

Therefore, with (2.13), we have

$$
\left|\left(\partial_{t}^{l} v_{0}, \Psi\right)\right| \leq C\left\|Q_{1} \phi_{1} w\right\|_{l+1, \Omega_{1}}+C\left\|Q_{2} \phi_{2} w\right\|_{l+1, \Omega_{1}}
$$

here the expression makes sense because the domain of dependence for $w$ and the pseudo-local properties of $Q_{1}$ and $Q_{2}$.

Now, we can apply Corollary 2.1 to obtain that

$$
\left\|Q_{2} w\right\|_{l+1, \Omega_{1}} \leq C\|\Psi\|_{0} .
$$

Lemma 2.3 yields

$$
\left\|Q_{2} w\right\|_{l+1, \Omega_{1}} \leq C\|\Psi\|_{0}
$$

where the constants here depend on $\left\|\sigma_{0}\right\|_{s}$.

Therefore, we have shown

$$
\left|\left(\partial_{l}^{l} v_{0}, \Psi\right)\right| \leq C\left\|\phi_{1} w\right\|_{l+1} \leq C\|\Psi\| .
$$

To complete the proof, we have one more step to go, that is, to estimate all the $x$ derivatives as well as the corresponding mixed derivatives of $v_{0}$ up to order of $l$. This may be done by introducing another dual problem. Here, we demonstrate this method by looking at $\partial_{x_{i}}^{l} v_{0}(i=1, \cdots, n)$. The rest terms can be estimated by the same fashion. It suffices to show that for any $\Psi \in C_{0}^{\infty}(\Omega)$

$$
\left|\left(\partial_{x_{i}}^{l} v_{0}, \Psi\right)\right| \leq C\|\Psi\|_{0}
$$

for $i=1, \cdots, n$.

Let $w$ solve the dual problem (2.9), $w_{1}$ solve the following problem

$$
\begin{aligned}
\square_{1} w_{1} & =\left[\frac{1}{\rho} \partial_{t}^{2}-\nabla \cdot\left(\frac{1}{\rho} \nabla\right)\right] w_{1}=\left[\partial_{x_{i}}^{l}, \square_{1}\right] w \\
w & =0 \quad t>T_{1} .
\end{aligned}
$$

We have

$$
\begin{aligned}
\left|\left(\partial_{x_{i}}^{l} v_{0}, \Psi\right)\right| & =\left|\left(v_{0}, \partial_{x_{i}}^{l} \square_{1} w\right)\right| \\
& \leq\left|\left(\square_{1} v_{0}, \partial_{x_{i}}^{l} w\right)\right|+\left|\left(\square_{1} v_{0}, w_{1}\right)\right| \\
& \leq C\left|\left(\partial_{x_{i}}^{l} \partial_{t}^{-(n-1) / 2} w\right)(0,0)\right|+C\left|\left(\partial_{t}^{-(n-1) / 2} w_{1}\right)(0,0)\right|
\end{aligned}
$$

Again, the trace theorem gives

$$
\begin{aligned}
\left|\left(\partial_{x_{i}}^{l} v_{0}, \Psi\right)\right| & \leq C\left\|\phi_{1} w\right\|_{l+1}+C\left\|\phi_{1} w_{1}\right\|_{\mathrm{I}} \\
& \leq C\left\|\phi_{1} w\right\|_{l+1}
\end{aligned}
$$

where in order to obtain the second inequality, we have applied the standard energy estimates and a commutator argument. From (2.17), the estimate (2.18) can then be proved. 


\section{Proof of the Main Theorem}

Our goal in this section is to determine the appropriate hypotheses under which $D F\left(\sigma_{0}\right)$, the linearization of $F$ about a reference state $\sigma_{0}$, is bounded. Our proof is based on the microlocal regularity analysis of the transport equations and regularity study of the dual problem.

Recall the linearized problem corresponding to the reference state $\left(u_{0}, \sigma_{0}\right)$, for $(t, x) \in$ $\mathbb{R}^{n+1}, x=\left(x^{\prime}, x_{n}\right)$,

$$
\begin{aligned}
& \left(\square-\nabla \sigma_{0} \cdot \nabla\right) \delta u=\nabla \delta \sigma \cdot \nabla u_{0} \\
& \delta u=0, \quad t<0,
\end{aligned}
$$

where $u_{0}$ is the solution of the model problem corresponding to the reference density $\sigma_{0}$. The linearized forward map can be defined as

$$
D F\left(\sigma_{0}\right) \delta \sigma=\left.(\phi \delta u)\right|_{x_{n}=0},
$$

where $\phi(x, t) \in C_{0}^{\infty}\left(\mathbb{R}^{n+1}\right)$ is supported inside the conoid $\{t>|x|\}$, and near $\left\{x_{n}=0\right\}$.

Once again we consider a related problem,

$$
\begin{aligned}
& \left(\square-\nabla \sigma_{0} \cdot \nabla\right) v=\nabla \delta \sigma \cdot \nabla v_{0} \\
& v=0, \quad t<0,
\end{aligned}
$$

where $\delta u=\partial_{t}^{\frac{n-1}{2}} v$ and $v_{0}$ solves

$$
\begin{aligned}
& \left(\square-\nabla \sigma_{0} \cdot \nabla\right) v_{0}=\delta^{-\frac{n-1}{2}}(t) \delta(x) \\
& v_{0}=0, \quad t<0 .
\end{aligned}
$$

Observe that for $l \in \mathbb{R}$,

$$
\begin{aligned}
\left\|D F\left(\sigma_{0}\right) \delta \sigma\right\|_{l} & =\left\|\left.(\phi \delta u)\right|_{x_{n}=0}\right\|_{l} \\
& \leq C\left\|\left.(\phi v)\right|_{x_{n}=0}\right\|_{l_{1}},
\end{aligned}
$$

where $l_{1}$ denotes $l+(n-1) / 2$. Thus the real challenge here is to get an appropriate trace regularity estimate for $v$ on a time-like hypersurface $\left\{x_{n}=0\right\}$.

Before getting into the details of the proof, let us first make the following general remarks on this theorem:

The estimate (1.8) has a similar form to a Rakesh's theorem (Theorem 2.5 in [18]). Actually, we conjecture that a formal extension of our proof here could lead to an elementary proof of his theorem. On the contrary, the principal tool of Rakesh's proof, calculus of Fourier integral operators, is not available when the reference density is nonsmooth.

Our approach enjoys the beauty of the method of energy estimates, that is, it possesses useful information on various parameters involved in the estimates. 


\subsection{Some useful results}

Hadamard's construction leads to the progressing wave expansion for $v_{0}$,

$$
v_{0}=\sum_{k=0}^{s} b_{k} S_{k}(t-\tau(x))+R_{v_{0}}(x, t)
$$

where $\tau(x)=|x|, S_{0}^{\prime}$ is the Heaviside function, $S_{k}^{\prime}=S_{k-1}(k \geq 1)$, and $R_{v_{0}}$ vanishes at $t=\tau(x)$. Moreover $\left\{b_{k}\right\}$ solve the transport equations, for $k=1, \cdots, s$,

$$
\begin{aligned}
& 2 \nabla \tau \cdot \nabla b_{0}+\left(\Delta \tau+\nabla \tau \cdot \nabla \sigma_{0}\right) b_{0}=0 \\
& 2 \nabla \tau \cdot \nabla b_{k}+\left(\Delta \tau+\nabla \tau \cdot \nabla \sigma_{0}\right) b_{k}=\Delta b_{k-1}+\nabla \sigma_{0} \cdot \nabla b_{k-1} .
\end{aligned}
$$

From (3.3), Hadamard's construction also gives the progressing wave expansion of $v$,

$$
v=\sum_{k=0}^{s} a_{k} S_{k}(t-\tau(x))+R_{v}(x, t),
$$

where $\tau(x)=|x|, S_{0}$ is the Heaviside function, $S_{k}^{\prime}=S_{k-1}, R_{v}$ vanishes at $t=\tau(x)$, and $\left\{a_{k}\right\}$ solve the transport equations, for $k=0, \cdots, s-1$,

$$
\begin{gathered}
2 \nabla \tau \cdot \nabla a_{0}+\left(\Delta \tau+\nabla \tau \cdot \nabla \sigma_{0}\right) a_{0}=-b_{0} \nabla \tau \cdot \nabla \delta \sigma \\
2 \nabla \tau \cdot \nabla a_{k+1}+\left(\Delta \tau+\nabla \tau \cdot \nabla \sigma_{0}\right) a_{k+1}=\Delta a_{k}+\nabla \sigma_{0} \cdot \nabla a_{k}+\nabla \delta \sigma\left(\nabla b_{k}-b_{k+1} \nabla \tau\right) .
\end{gathered}
$$

In fact, the equations (3.10) and (3.11) are the first order perturbation of the equations (3.7) and (3.8), respectively.

For convenience, we introduce a function $q=\sigma_{0} / 2+q_{0}$ with $\nabla \tau \cdot \nabla q_{0}=\Delta \tau / 2$. Thus away from the origin, $q$ is nothing more than a smooth perturbation of $\sigma_{0} / 2$. Then the transport equations $(3.7),(3.8)$ may be transformed to equations

$$
\begin{aligned}
& \nabla \tau \cdot \nabla b_{0} \epsilon^{q}=0 \\
& \nabla \tau \cdot \nabla b_{k} \epsilon^{q}=\left(\Delta b_{k-1} / 2+\nabla \sigma_{0} \cdot \nabla b_{k-1} / 2\right) e^{q},
\end{aligned}
$$

for $k=1, \cdots, l_{1}$.

Similarly, equations (3.10) and (3.11) may be transformed to equations

$$
\begin{aligned}
\nabla \tau \cdot \nabla a_{0} e^{q} & =\nabla \tau \cdot \nabla\left(\delta \sigma b_{0} e^{q}\right) \\
\nabla \tau \cdot \nabla a_{k} e^{q} & =\left(\Delta a_{k-1} / 2+\nabla \sigma_{0} \cdot \nabla a_{k-1} / 2\right) e^{q}+\nabla \delta \sigma\left(\nabla b_{k}-b_{k+1} \nabla \tau\right) e^{q} / 2
\end{aligned}
$$

for $k=1, \cdots, l_{1}$, where to obtain the first equation, we have used the equation $\nabla \tau \cdot \nabla b_{0} e^{q}=0$. Observation. The right hand side of the equation (3.13) may be rewritten in terms of $b_{k-1} e^{q}$ as

$$
\Delta\left(b_{k-1} e^{q}\right) / 2-|\nabla q|^{2} b_{k-1} e^{q} / 2-b_{k-1} e^{q} \Delta q / 2-\nabla\left(b_{k-1} e^{q}\right) \cdot \nabla q_{0}+b_{k-1} e^{q} \nabla q \cdot \nabla q_{0} .
$$

Similar observation may be made for the right hand side of (3.15). 
Observe that all the transport equations have the same principal part $\nabla \tau \cdot \nabla$ which is a smooth vector field. Therefore, in order to understand the regularity of the solutions to (3.12)-(3.15) it is essential to study the properties of this smooth vector field. The following is a microlocal regularity result for the solutions of transport equations. It indicates that a refined regularity result can be achieved microlocally away from the characteristic variety of the wave operator, in contrast to a direct application of the energy estimates. This result will be used frequently throughout the proof of Theorem 1.1.

Lemma 3.1 Let $V$ be a smooth vector field. Assume that $u$ is a smooth function, $Q \in O P S^{0}$ supported away from Char $(V)$, and $\phi \in C_{0}^{\infty}(\Omega)$ where $\Omega$ is a bounded open set. Then there exist $Q^{\prime} \in O P S^{0}$ also supported away from $C h a r(V)$ and $\phi^{\prime}, \bar{\phi} \in C_{0}^{\infty}\left(\Omega^{\prime}\right)$ with $\Omega \subset \subset \Omega^{\prime}$, such that for any $r \in \mathbb{R}$,

$$
\|Q \phi u\|_{s, \Omega} \leq C\left\|Q^{\prime} \phi^{\prime} V u\right\|_{s-1, \Omega^{\prime}}+C\|\bar{\phi} u\|_{r, \Omega^{\prime}}
$$

Proof. Let $Q_{1}$ be another $\psi$.d.o. of order zero, such that $Q_{1}$ is elliptic on $E S(Q)$ and $E S\left(Q_{1}\right)$ is away from $\operatorname{Char}(V)$. It follows from Lemma 2.1 that there exists $\Omega_{1} \supset \supset \Omega$ an open bounded set such that

$$
\begin{aligned}
\|Q \phi u\|_{s, \Omega} & \leq C\left\|Q_{1} V \phi u\right\|_{s, \Omega_{1}}+C\|\phi u\|_{r, \Omega_{1}} \\
& \leq C\left\|Q_{1}[V, \phi] u\right\|_{s-1, \Omega_{1}}+C\left\|Q_{1} \phi V u\right\|_{s-1, \Omega_{1}}+C\|\phi u\|_{r, \Omega_{1}} .
\end{aligned}
$$

Now choose $\phi_{1} \in C_{0}^{\infty}\left(\Omega_{1}\right)$ and $\phi_{1}=1$ on supp $(\phi)$, then

$$
Q_{1}[V, \phi] u=Q_{1}[V, \phi] \phi_{1} u=\tilde{Q}_{1} \phi_{1} u
$$

where $\tilde{Q}_{1}=Q_{1}[V, \phi]$ is a $\psi$.d.o. of order zero and $E S\left(\tilde{Q}_{1}\right)$ is away from $C h a r(V)$.

Thus

$$
\|Q \phi u\|_{s, \Omega} \leq C\left\|\dot{Q}_{1} \phi_{1} u\right\|_{s-1, \Omega_{1}}+C\left\|Q_{1} \phi V u\right\|_{s-1, \Omega_{1}}+C\|\phi u\|_{r, \Omega_{1}}
$$

We can then repeat the above process to get a similar estimate on $\left\|\tilde{Q}_{1} \phi_{1} u\right\|_{s-1, \Omega_{1}}$. In fact, one can construct: $Q_{2} \in O P S^{\circ}, Q_{2}$ is elliptic on $E S\left(\tilde{Q}_{1}\right)$ and $E S\left(Q_{2}\right)$ is away from $C h a r(V)$; $\phi_{2} \in C_{0}^{\infty}\left(\Omega_{2}\right), \phi_{2}=1$ on $\operatorname{supp}(\phi), \Omega_{1} \subset \subset \Omega_{2} ; \tilde{Q}_{2}=Q_{2}\left[V, \phi_{1}\right]$.

Then

$$
\left\|\tilde{Q}_{1} \phi_{1} u\right\|_{s-1, \Omega_{3}} \leq C\left\|\dot{Q}_{2} \phi_{2} u\right\|_{s-2, \Omega_{2}}+C\left\|Q_{2} \phi_{1} V u\right\|_{s-2, \Omega_{2}}+C\left\|\phi_{1} u\right\|_{r, \Omega_{2}} .
$$

Following the same idea, one may lieep going to get similar estimates: (the $i$-th step) Construct: $Q_{i} \in O P S^{0}, Q_{i}$ is elliptic on $E S\left(Q_{i-1}\right)$ and $E S\left(Q_{i}\right)$ is away from $C h a r(V)$; $\phi_{i} \in C_{0}^{\infty}\left(\Omega_{i}\right), \phi_{i}=1$ on $\operatorname{supp}\left(\phi_{i-1}\right) . \Omega_{i-1} \subset \subset \Omega_{i} ; \tilde{Q}_{i}=Q_{i}\left[V, \phi_{i}\right], 0 \leq i \leq k-1$. $(k$ is chosen to satisfy $s-k \leq r)$.

Then

$$
\left\|\dot{Q}_{i} \phi_{i} u\right\|_{s-i, \Omega_{i}} \leq C\left\|\dot{Q}_{i+1} \phi_{i+1} u\right\|_{s-i-1, \Omega_{i+1}}+C\left\|Q_{i+1} \phi_{i} V u\right\|_{s-i-1, \Omega_{i+1}}+C\left\|\phi_{i} u\right\|_{r, \Omega_{i+1}} .
$$


Combining all of the estimates above, we have

$$
\|Q \phi u\|_{s, \Omega} \leq C\left\|\tilde{Q}_{k} \phi_{k} u\right\|_{s-k, \Omega_{k}}+C \sum_{i=0}^{k-1}\left\|Q_{i+1} \phi_{i} V u\right\|_{s-i-1, \Omega_{i+1}}+C \sum_{i=0}^{k-1}\left\|\phi_{i} u\right\|_{r, \Omega_{i+1}}
$$

In order to get the desired estimate, some further simplifications are needed. Clearly, for $j=0, \cdots, k-2$,

$$
\left\|\phi_{j} u\right\|_{r, \Omega_{j+1}} \leq C\left\|\phi_{j+1} u\right\|_{r, \Omega_{j+2}}
$$

hence

$$
\sum_{i=0}^{k-1} C\left\|\phi_{i} u\right\|_{r, \Omega_{i+1}} \leq C\left\|\phi_{k-1} u\right\|_{r, \Omega_{k}}
$$

Since $s-k \leq r,\left\|\dot{Q}_{k} \phi_{k} u\right\|_{s-k, \Omega_{k}} \leq C\left\|\phi_{k} u\right\|_{r, \Omega_{k}}$, therefore,

$$
\|Q \phi u\|_{s, \Omega} \leq C\left\|\phi_{k} u\right\|_{r, \Omega_{k}}+\sum_{i=0}^{k-1}\left\|Q_{i+1} \phi_{i} V u\right\|_{s-i-1, \Omega_{i+1}} .
$$

Construct a $\psi$.d.o. of order zero, $Q^{\prime}$, with the following properties:

$Q^{\prime}$ is elliptic on $\cup_{i=0}^{k-1} E S\left(Q_{i+1} \phi_{i}\right) ; E S\left(Q^{\prime}\right)$ is away from $C h a r(V)$. Lemma 2.1 then yields that

$$
\begin{aligned}
\left\|Q_{i+1} \phi_{i} V u\right\|_{s-1, \Omega_{k}} & =\left\|Q_{i+1} \phi_{i} \phi_{k} V u\right\|_{s-1, \Omega_{k}} \\
& \leq C\left\|Q^{\prime} \phi_{k} V u\right\|_{s-1, \Omega^{\prime}}+C\left\|\phi_{k} V u\right\|_{r-1, \Omega^{\prime}}
\end{aligned}
$$

where $\Omega_{k} \subset \subset \Omega^{\prime}, i=0,1, \cdots, k-1$. Therefore, if we construct $\bar{\phi} \in C_{0}^{\infty}\left(\Omega^{\prime}\right)$ in such a way that $\operatorname{ar} \phi>0$ on $\operatorname{supp}\left(\phi_{k}\right)$, then

$$
\left\|\phi_{k} V u\right\|_{r-1, \Omega^{\prime}} \leq C\|\bar{\phi} u\|_{r, \Omega^{\prime}}
$$

Let $\phi^{\prime}$ be $\phi_{k}$, we finally obtain

$$
\|Q \phi u\|_{s, \Omega} \leq C\left\|Q^{\prime} \phi^{\prime} V u\right\|_{s-1, \Omega^{\prime}}+C\|\bar{\phi} u\|_{r, \Omega^{\prime}}
$$

which completes our proof.

We also need the following standard result for hyperbolic p.d.e, as well as the estimates involving in its proof. See, for example, Chazarain-Piriou [9] for the idea of the proof. The following is the version stated in Beals [5].

Lemma 3.2 (Linear Energy Inequality) Let $p(x, D)$ be a partial differential operator of order $m$ on $\mathbb{R}^{n+1}$, strictly hyperbolic with respect to the plane $\left\{x_{n+1}=0\right\}$, and let $u$ satisfy $p(x, D) u=f(x)$. If $f \in H_{l o c}^{s-m+1}\left(\mathbb{R}^{n+1}\right)$ and $u \in H_{l o c}^{s}\left(x:\left|x_{n+1}\right| \leq \epsilon\right)$ for some $\epsilon>0$, then $u \in H_{l o c}^{s}\left(\mathbb{R}^{n+1}\right)$.

Next, we present a trace regularity result. 
Lemma 3.3 Assume that $s>3+n / 2,1 \leq l_{1} \leq s$, and $v$ solves problem (3.3) then there is $a \phi_{0} \in C_{0}^{\infty}$ supported near supp $(\phi)$ such that the following estimate holds,

$$
\left\|\left.(o v)\right|_{n_{n}=0}\right\|_{l_{1}} \leq C\left\|\phi_{0} v\right\|_{l_{1}}
$$

where $C$ is a constant depending on the $H^{s} \cap H_{m \ell}^{l_{1}+1}(K)$-norm of $\sigma_{0}$, but is independent of $\delta \sigma$.

Proof. This lemma is a direct application of Theorem 3.1 in [3] by taking into account of the fact that $\phi$ and $\delta \sigma$ have disjoint supports.

\subsection{Microlocal version of trace theorem}

The classical trace theorem in Sobolev spaces characterizes the regularity of a distribution restricted to a hypersurface. Dealing with inverse problems, one always has to face a difficult but crucial question: When does the restriction operator commute with another operator of interest? The result in this subsection indicates that a simple microlocal trace theorem, which not only works on the space restriction but also on the phase restriction (i.e. a trace theorem on cotangent bundles), may lead to a way to cure this difficulty.

Let $K$ be a conic set in $\mathbb{R}^{n}, i: x \in \mathbb{R}^{n} \rightarrow(x, 0) \in \mathbb{R}^{n+1}$. Define a semi-norm: for $\gamma$ a conic set of $\mathbb{R}^{k}$ and $u \in C_{0}^{\infty}\left(\mathbb{R}^{k}\right)$,

$$
|u|_{\gamma, i}=\left(\int_{\xi \in \gamma} d \xi|\hat{u}(\xi)|^{2}\langle\xi\rangle^{2 s}\right)^{1 / 2}
$$

Then, a proof of the classical trace theorem (see e.g. in Taylor [26], pages 20-21) implies the following inequality.

Proposition 3.1 For $s>1 / 2, u \in C_{0}^{\infty}\left(\mathbb{R}^{n+1}\right)$,

$$
\left|i^{*} u\right|_{K, s-1 / 2} \leq C|u|_{K \times \mathbb{R}, s} \text {. }
$$

Thus the map $i^{\star}$ may be extended to be a bounded map from $H_{m \ell}^{s}(x \times \mathbb{R}, K \times \mathbb{R})$ to $H_{m \ell}^{s-1 / 2}(x, K)$, provided $s>1 / 2$.

Let $\Pi_{2}$ be the projection map to the frequency space (or the second factor). We may reformulate this result in terms of t.d.o..

Proposition 3.2 If $P_{1}$ is a $\psi$.d.o. of order zero in $\mathbb{R}^{n}$, with $\Pi_{2} E S\left(P_{1}\right) \subset K$, then there exists a $\psi$.d.o. $P_{2}$ of order zero in $\mathbb{R}^{n+1}$, and $\Pi_{2} E S\left(P_{2}\right) \subset K \times \mathbb{R}$, such that for $s>1 / 2$, $u \in C_{0}^{\infty}(\Omega)$ with $\Omega$ an open bounded subset of $\mathbb{R}^{n+1}$,

$$
\left\|P_{1} i^{*} u\right\|_{s-1 / 2, \Omega_{0}} \leq C\left\|P_{2} u\right\|_{s, \Omega},
$$

where $i^{*}$ again denotes a restriction operator to a codimension one hypersurface and $\Omega_{0}=$ $i^{*} \Omega$. 
The above results together with our Gàrding's type result Lemma 2.1 yield a microlocal version of trace theorem.

Lemma 3.4 Assume that $E$ is an elliptic operator of order $m$ in $\mathbb{R}^{n+1} \times K \times \mathbb{R}, P \in$ $O P S^{0}\left(\mathbb{R}^{n}\right)$ and $\Pi_{2} E S(P) \subset K$. Then for $s>1 / 2, u \in C_{0}^{\infty}(\Omega)$ where $\Omega$ and $\Omega^{\prime}$ are open bounded subsets of $\mathbb{R}^{n+1}$ with $\Omega \subset \subset \Omega^{\prime}$, and $\Omega_{0}=i^{*} \Omega$,

$$
\left\|P i^{*} u\right\|_{s-1 / 2, \Omega_{0}} \leq C\|E u\|_{s-m, \Omega^{\prime}}+C\|u\|_{r, \Omega^{\prime}}
$$

for any $r \in \mathbb{R}$.

A result on propagation of singularities, see Proposition 1.3.3 in Duistermaat [11] or Theorem 8.2.13 in Hörmander [11], demonstrates the relation between the wavefront of the restriction of a distribution and the wavefront set of its own. However the result does not directly lead to any explicit bound. Here, in this direction, we present a result together with an estimate. The proof follows directly from Lemma 2.2 and Lemma 2.3.

Lemma 3.5 Assume that $s>n / 2, \sigma_{0} \in H^{s+1}\left(\mathbb{R}^{n}\right)$. Suppose that $w$ is smooth near $\{t=0\}$ and $\square w-\nabla \sigma_{0} \cdot \nabla w \in L^{2}\left(\mathbb{R}^{n+1}\right)$. Then there exists an elliptic $\psi . d . o . \tilde{B}$ of order zero, such that $\operatorname{ES}(\hat{B})$ is contained in $C y$, a "cylindrical" conic neighborhood of

$$
\left\{(x, t, \xi, \omega) \in T^{*} \mathbb{R}^{n+1} \backslash 0, t^{2}-|x|^{2}=0, \omega=\nabla \tau \cdot \xi\right\}
$$

along $\omega$ direction, and the symbol of $\dot{B}$. i satisfies

$$
\Pi \operatorname{supp}(\dot{b}) \cap \operatorname{supp}\left(\square w-\nabla \sigma_{0} \cdot \nabla w\right)=\emptyset .
$$

Then, for any $\phi_{1} \in C_{0}^{\infty}\left(\mathbb{I R}^{n+1}\right)$ and $k<s-n / 2+2$,

$$
\left\|\phi_{1} \tilde{B} w\right\|_{k} \leq C\left\|\square w-\nabla \sigma_{0} \cdot \nabla w\right\|_{0}
$$

where the constant $C$ depends on $\sigma_{0}, k, \dot{B}$, and $\phi_{1}$, but not on $w$.

\subsection{Proof of Theorem 1.1}

Recall the assumption made in Section 1.

(A) $\operatorname{supp}(\delta \sigma) \subset\left\{x_{n}>\epsilon\right\}$,

for $\epsilon>0$ small.

For simplicity, we shall also assume that $l_{1}$ is an integer. Without any further difficulty, the proof may be extended formally to cover the general case.

Let $\phi \in C_{0}^{\infty}$ be supported insicle the characteristic surface and the set $\left\{x_{n}<\epsilon / 2\right\}$. Multiplying $\phi$ to both sides of equation (3.3), we have

$$
\begin{gathered}
\square \phi v=\phi \nabla \sigma_{0} \cdot \nabla v+[\square, \phi] v \\
v=0, \quad t<0 .
\end{gathered}
$$


Here we have used the fact that according to the assumption (A), $\phi$ and $\delta \sigma$ have disjoint supports, so that $\phi \nabla \delta \sigma \cdot \nabla v_{0}=0$.

Having Lemma 3.3 , the estimate of $\left\|\left.(\phi v)\right|_{x_{n}=0}\right\|_{l_{1}}$ may be reduced to estimating $\left\|\phi_{0} v\right\|_{l_{1}}$. For the sake of simplicity, we shall only discuss the estimates of the $t$-derivatives of $v$. The rest may be estimated as in the proof of Theorem 2.1 .

The rest of this section is devoted to the estimate of $\left\|\phi_{0} v\right\|_{l_{1}}$.

We study the regularity of $v$ through its dual problem. Once again, we look at the symmetric form, for $\rho(x)=e^{-\sigma_{0}}$.

$$
\begin{aligned}
\square_{1} v & =\left[\frac{1}{\rho} \partial_{t}^{2}-\nabla \cdot\left(\frac{1}{\rho} \nabla\right)\right] v_{0}=\frac{1}{\rho} \nabla \delta \sigma \cdot \nabla v_{0} \\
v & =0 \quad t<0 .
\end{aligned}
$$

A clual problem to $(3.24)$,

$$
\begin{aligned}
\square_{1}^{\prime} w & =\left[\frac{1}{\rho} \partial_{t}^{2}-\nabla \cdot\left(\frac{1}{\rho} \nabla\right)\right] w=\Psi \\
w & =0 \quad t>>T_{1},
\end{aligned}
$$

where $\Psi \in C_{0}^{\infty}(\Omega)$ with $\Omega=\left\{\mathbb{R}^{n} \times\left(0, T_{1}\right)\right\} \cap\{t>|x|+\epsilon\}$, for a small $\epsilon>0$.

Thus if we can show that for any $\Psi \in C_{0}^{\infty}(\Omega)$

$$
\left|\left(\partial_{t}^{l_{1}} v_{2}, \Psi\right)\right| \leq C\|\delta \sigma\|_{l_{1}}\|\Psi\|_{0}
$$

then it can be concluded that

$$
\left\|\partial_{t}^{l_{1}} v_{2}\right\|_{0, \Omega} \leq C\|\delta \sigma\|_{l_{1}}
$$

Green's identity and integration by parts lead to

$$
\begin{aligned}
& \left(\partial_{t}^{l_{1}} v, \Psi\right)=\left(\partial_{t}^{l_{1}} v, \square_{1}^{\prime} w\right) \\
& =\left(\square_{1} \partial_{t}^{l_{1}} v, w\right)-2 \int_{t=\tau(x)} \frac{1}{\rho}\left[\partial_{t}^{l_{1}} v \frac{\partial}{\partial n} w-w \frac{\partial}{\partial n} \partial_{t}^{l_{1}} v\right] d s
\end{aligned}
$$

Let us look at the first term. From equation (3.3),

$$
\left(\square_{1} \partial_{t}^{l_{1}} v, w\right)=\left(\nabla \delta \sigma \cdot \nabla \partial_{t}^{l_{1}} v_{0}, w\right)
$$

Thus

$$
\left|\left(\square_{1} \partial_{t}^{l_{1}} v, w\right)\right| \leq C\|\delta \sigma\|\left\|\partial_{t}^{l_{1}} v_{0}\right\|_{0, \Omega}\|w\|_{1, \Omega} .
$$

Applying Theorem 2.1 and energy estimates to the right hand side, we have

$$
\left|\left(\square_{1} \partial_{t}^{l_{1}} v, w\right)\right| \leq C\|\delta \sigma\|\|\Psi\|
$$

with the constant $C$ depending on $\left\|\sigma_{0}\right\|_{s}$, for $s>\max \left\{1+n / 2, l_{1}+n / 2\right\}$.

Using Hardamard's construction, one may get:

$\left.\left(\partial_{t}^{l_{1}} v\right)\right|_{t=\tau(x)}=a_{l_{1}},\left.\left(\partial_{t}^{l_{1}+1} v\right)\right|_{t=\tau(x)}=a_{l_{1}+1},\left.\left(\partial_{x} \partial_{t}^{l_{1}} v\right)\right|_{t=\tau(x)}=\partial_{x} a_{l_{1}}+a_{l_{1}+1}$. 
Denote

$$
I_{1}=\int_{t=\tau(x)} \frac{1}{\rho} \partial_{t}^{l_{1}} v \frac{\partial}{\partial n} w d s, \quad I_{2}=\int_{t=r(x)} \frac{1}{\rho} \frac{\partial}{\partial n}\left(\partial_{t}^{l_{1}} v\right) w d s .
$$

Observation. The integrands are all compactly supported even though neither $v$ nor $w$ is compactly supported.

Since the above integrals lrave similar forms, we will only give a detail estimate of $I_{1}$ by making the following remark on the estimate of $I_{2}$ :

$$
I_{2}=-2 \int_{t=\tau(x)} \frac{1}{\rho} \nabla \tau \cdot \nabla\left(\left.\partial_{t}^{l_{1}} v\right|_{t=\tau}\right) w d s+\int_{t=\tau(x)} \frac{1}{\rho} \partial_{t}^{l_{1}+1} v w d s .
$$

Now, by integration by parts, the first term of the right hand side can be studied just like $I_{1}$; while the second term may also be handled in a similar fashion as for $I_{1}$.

\subsection{The estimate of $I_{1}$}

A function $\phi(x) \in C_{0}^{+\infty}(\Omega), \Omega$ an open bounded set in $\mathbb{R}^{n}$ and $\Omega \supset \operatorname{supp}\left\{\left.\left.\partial_{t}^{l} v\right|_{t=\tau} \frac{\partial}{\partial n} w\right|_{t=\tau}\right\}$, may be found because the integrand of $I_{1}$ is compactly supported, so that

$$
I_{1}=\int_{l=\tau(s)} \phi \frac{1}{\rho} \partial_{t}^{l} v \frac{\partial}{\partial n} w d s
$$

Now construct two $\psi$.d.o. $Q_{1}, Q_{2} \in O P S^{0}\left(\mathrm{IR}^{n}\right)$

- $Q_{1}+Q_{2}=I$

- $E S\left(Q_{2}\right)$ is a small conic neighborhood of $\{\nabla \tau \cdot \xi=0\}$;

- $Q_{2}$ 's symbol $q_{2}=1$ near $\{\nabla \tau \cdot \xi=0\} \cap\{(x, \xi),|\xi| \geq 1\}$.

Recall the definitions of $Q_{1}$ and $Q_{2}$. We can again rewrite $I_{1}$ as, for a $\phi_{1}(x) \in C_{0}^{\infty}\left(\Omega^{\prime}\right)$ with $\Omega \subset \Omega^{\prime}$

$$
\begin{aligned}
I_{1} & =\int_{t=\tau} Q_{1} \phi \frac{1}{\rho} \partial_{t}^{l_{1}} v Q_{1} \phi_{1} \frac{\partial}{\partial n} w d s+\int_{t=\tau} Q_{1} \phi \frac{1}{\rho} \partial_{t}^{l_{1}} v Q_{2} \phi_{1} \frac{\partial}{\partial n} w d s+\int_{t=\tau} Q_{2} \phi \frac{1}{\rho} \partial_{t}^{l_{1}} v \phi_{1} \frac{\partial}{\partial n} w d s \\
& =I_{1, a}+I_{1, b}+I_{1, c} .
\end{aligned}
$$

Now, we estimate $I_{1, a}, I_{1, b}$, and $I_{1, c}$ separately.

(a) The estimate of $I_{1, a}$

Clearly, in order to estimate $I_{1, a}$, it suffices to estimate $\left\|Q_{1} \phi \frac{1}{\rho} a_{l_{1}}\right\|$ and $\left\|\left.Q_{1} \phi_{1} \frac{\partial}{\partial n} w\right|_{t=\tau}\right\|$. These terms may be handled by the following propositions.

Proposition 3.3 The following cstimale holds

$$
\left\|Q_{1} \phi b_{k} e^{q}\right\|_{0, \Omega} \leq C_{k}
$$

where $\Omega \subset \subset \grave{\Omega}$ and $C_{k}$ depends on $\left\|\sigma_{0}\right\|_{k}$. 
Proof. The proof is based on an application of Lemma 3.2. We also have to use variants of Rauch's Lemma, Schauder's Lemma, together with Proposition 2.1.

In fact, Lemma 2.2 leads to, for any $r$,

$$
\left\|Q_{1} \phi b_{k} e^{q}\right\|_{0, \Omega} \leq C\left\|Q_{1}^{\prime} \phi_{1}^{\prime} \nabla \tau \cdot \nabla b_{k} e^{q}\right\|_{-1, \Omega_{1}}+C\left\|\bar{\phi}_{1} b_{k} e^{q}\right\|_{r, \Omega_{1}}
$$

where $\Omega \subset \subset \Omega_{1}, \phi_{1}^{\prime}, \bar{\phi}_{1} \in C_{0}^{\infty}\left(\Omega_{1}\right)$, and $Q^{\prime}$ supported away from $C h a r(\nabla \tau \cdot \nabla)$. From (3.13), it is easy to represent the right hand side in terms of $b_{k-1} e^{q}$. Therefore, Rauch's Lemma and Proposition 2.1 yield

$$
\left\|Q_{1} \phi b_{k} e^{q}\right\|_{0 . \Omega} \leq C\left\|Q_{1}^{\prime} \phi_{2} b_{k-1} e^{q}\right\|_{1, \Omega_{1}}+C\left\|\bar{\phi}_{1} b_{k}\right\|_{r, \Omega_{1}}
$$

where $s>1+n / 2$ and the constant $C$ depends on $\left\|\sigma_{0}\right\|_{s}$.

One can then continue this process by applying Lemma 2.2 to estimate $\left\|Q_{1}^{\prime} \phi_{2} b_{k-1} e^{q}\right\|_{1, \Omega_{1}}$. After $l$ similar steps, we can write the following estimate, for any $r$,

$$
\left\|Q_{1} \phi b_{k} e^{q}\right\|_{0, \Omega} \leq C\left\|Q_{l}^{\prime} \phi_{l} b_{0} e^{q}\right\|_{k, \Omega_{l}}+\sum_{i=1}^{k} C\left\|\bar{\phi}_{i} b_{i}\right\|_{r, \Omega_{i}}
$$

where $C$ depends on $\left\|\sigma_{0}\right\|_{s}$ for $s>1+n / 2, \Omega \subset \subset \Omega_{1} \subset \subset \cdots \subset \subset \Omega_{k}$, and $\bar{\phi}_{i} \in C_{0}^{\infty}\left(\Omega_{i}\right)$.

We next discuss why the smoothing terms $\left\|\bar{\phi}_{i} b_{i}\right\|_{r, \Omega_{i}}$ may be negligible.

Note that the transport equations are hyperbolic along the $\lambda$ direction $(\lambda=|x|)$. Actually, introducing polar coordinates, we then get $\nabla \tau \cdot \nabla=\frac{\partial}{\partial \lambda}(\lambda=|x|)$. Thus, $\lambda$ may be treated as the "time" variable for a standard hyperbolic problem. The right hand sides of transport equations may be expressed under the polar coordinates as

$$
\frac{d b_{k} e^{q}}{d \lambda}
$$

Because of the main assumption, $\sigma(x)$ is constant near $|x|=0$, that is, $v_{0}$ is the fundamental solution for the same equation with constant coefficients. The Hadamard construction yields that $b_{k}\left(k=0, \cdots, l_{1}\right)$ are smootl function near $|x|=0$ (or $\left.\lambda=0\right)$, see [20] or [1] for more discussions. Lemma 3.2 then becomes applicable.

Eventually, there exists a constant $C_{k}$ depending on $\left\|\sigma_{0}\right\|_{k}$, so that

$$
\left\|Q_{1} \phi b_{k} \epsilon^{q}\right\| \leq C_{k}
$$

Proposition 3.4 The following estimate holds

$$
\left\|Q_{1} \phi a_{l_{1}} e^{q}\right\|_{0, \Omega} \leq C\|\delta \sigma\|_{l_{1}}
$$

where $C$ depends on $\left\|\sigma_{0}\right\|_{s}$ for $s>\left\{1+n / 2, l_{1}\right\}$. 
Remark. With a few necessary changes, the proof of Proposition 3.3will still work. Observe that the only major difference to the analysis before is that each one of the transport equations now possesses an extra term, which may be viewed as a "source" term. Certainly, these extra terms are responsible for the $\delta \sigma$ term in the estimate of our main theorem.

We may also rewrite the right hand side of (3.15) in terms of $a_{k-1} e^{q}$ plus a source term containing $\delta \sigma$. The desired estimate then follows by applying Lemma 3.4, together with algebraic estimates, various forms of Schauder's Lemma, Rauch's Lemma Proposition 2.1, and commutator lemmas.

Note that the transport equations of $a_{k}$ may be viewed as the first order perturbation of the corresponding equations of $b_{k}$. Hence near $|x|=0$, by the main assumption $(A) \delta \sigma=0$, $a_{k}=0$ for $k=1, \cdots, l_{1}$.

Next, we want to get an estimate of $\left\|\left.Q_{1} \phi_{1} \frac{\partial}{\partial n} w\right|_{t=r}\right\|_{0, \Omega^{\prime}}$.

Proposition 3.5 For $Q_{1}, w, \Psi$, and $\phi_{1}$ as previously defined, we have

$$
\left\|\left.Q_{1} \phi_{1} \frac{\partial}{\partial n} w\right|_{t=\tau}\right\|_{0, \Omega^{\prime}} \leq C\|\Psi\|
$$

where the constant $C$ depends on $\left\|\sigma_{0}\right\|_{s}$, for $s>1+n / 2$.

Proof. Since $w$ solves the dual problem (2.9), which has smooth right hand side, we need a characteristic trace regularity result for $w$. Note that $E S\left(Q_{1}\right)$ is away from the characteristic variety of the wave equation. In lact, an application of a characteristic trace regularity result, Corollary 2 in [2], yields that there exists a $\dot{\phi} \in C_{0}^{\infty}\left(\mathbb{R}^{n+1}\right)$, such that

$$
\left\|\left.Q_{1} \phi_{1} \frac{\partial}{\partial n} w\right|_{t=r}\right\|_{0, \Omega^{\prime}} \leq C\|\tilde{\phi} w\|_{1} \leq C\|\Psi\|
$$

the second inequality comes from the standard energy estimates.

(b). The estimate of $I_{1, b}$

Because of Proposition 3.4, in order to bound $I_{1, b}$, it suffices to estimate $\left.|| Q_{2} \phi_{1} \frac{\partial}{\partial n} w\right|_{t=\tau} \|_{0, \Omega^{\prime}}$. This requires the microlocal trace regularity result Lemma 3.4 and the regularity analysis of the dual problem Lemma 3.5. The estimate can be easily derived by applying Lemma 3.4 and Lemma 3.5 .

Proposition 3.6 For $Q_{2}, w$, and $\phi_{1}$ defined above. The following holds

$$
\left\|\left.Q_{2} \phi_{1} \frac{\partial}{\partial n} w\right|_{t=\tau}\right\|_{0, \Omega^{\prime}} \leq C\|\Psi\|
$$

with the constant $C$ depending on $\left\|\sigma_{0}\right\|_{s}$ for $s>1+n / 2$

(c). The estimate of $I_{1, \mathrm{c}}$

We want to estimate

$$
\int_{t=T(x)} Q_{2} \phi \frac{1}{\rho} a_{l_{1}} \phi_{1} \frac{\partial}{\partial n} w d s .
$$


Obviously, this term has to be handled very differently. In fact, the transport operator (the operator appears in the transport cquations) is elliptic on $E S\left(Q_{1}\right)$, by Lemma 3.1 the $L^{2}$ norm of $Q_{1} \phi a_{l_{1}}$ may be bounded by the $H^{l_{1}}$ norm of $Q_{1} \phi a_{0}$. However, there is no ellipticity on $E S\left(Q_{2}\right)$, and the only direct estimate one may expect is by applying Lemma 3.2, which implies that essentially the $L^{2}$ norm of $Q_{2} \phi a_{l_{1}}$ has to be bounded by the $H^{2 l_{1}}$ norm of $\phi a_{0}$.

To cure this difficulty, we introduce a "bootstrap" argument by taking advantage of the transport equations and the fact that $\left.Q_{2} \phi_{1} \frac{\partial}{\partial n} w\right|_{t=\tau}$ is a much smoother term (it would be smooth if $\sigma_{0}$ were smooth). Again, we start with the corresponding estimate for $b_{l_{1}}$.

Proposition 3.7 The estimate

$$
\left|\int_{t=r(x)} Q_{2} \phi \frac{1}{\rho} b_{l_{1}} \phi_{1} \frac{\partial}{\partial n} w d s\right| \leq C\|\Psi\|
$$

holds with the constant $C$ depending on $\left\|\sigma_{0}\right\|_{s}$, for $s>l_{1}+(n-3) / 2$.

Proof. Without loss of generalities, $Q_{2}$ may be assumed to be self-adjoint. Thus

$$
\int_{t=\tau(x)} Q_{2} \phi \frac{1}{\rho} b_{l_{1}} \phi_{1} \frac{\partial}{\partial n} w d s=\int_{t=\tau(x)} \frac{1}{\rho} b_{l_{1}} \phi Q_{2} \phi_{1} \frac{\partial}{\partial n} w d s .
$$

Since $Q_{2} \phi_{1} \frac{\partial}{\partial n} w$ is much smoother than the other part, one wants to apply transport equations to reduce $b_{l_{1}}$ to the one requires the least regularity.

Consider a problem

$$
\begin{aligned}
(\nabla T \cdot \nabla)^{*} W_{1} & =\left.\phi e^{\sigma_{0}-q} Q_{2} \phi_{1}\left(\frac{\partial}{\partial n} w\right)\right|_{t=\tau(x)} \\
W_{1} & =0 \text { near }|x|=0
\end{aligned}
$$

Then (3.33) becomes

$$
\int_{t=\tau(x)} Q_{2} \phi \frac{1}{\rho} b_{1} \phi_{1} \frac{\partial}{\partial n} w d s=\int_{t=\tau(x)} \nabla \tau \cdot \nabla b_{l_{1}} e^{q} W_{1} d s
$$

From the transport equation (3.16), we have

$$
\begin{aligned}
\int_{t=\tau(x)} \nabla \tau \cdot \nabla b_{l_{1}} e^{q} W_{1} d s= & \int_{t=\tau(x)}\left(\Delta\left(b_{l_{1}-1} e^{q}\right) / 2-|\nabla q|^{2} b_{l_{1}-1} e^{q} / 2-\Delta q b_{l_{1}-1} e^{q} / 2\right. \\
& \left.-\nabla\left(b_{l_{1}-1} e^{q}\right) \nabla q_{0}+b_{l_{1}-1} e^{q} \nabla q \nabla q_{0}\right) W_{1} d s \\
= & \int_{t=\tau(x)} b_{l_{1}-1} e^{q} P_{1} W_{1} d s
\end{aligned}
$$

where $P=\Delta-|\nabla q|^{2}-\Delta q+\nabla \cdot \nabla \sigma_{0}+\nabla q \cdot \nabla q_{0}$.

One can then introduce another problem

$$
\begin{aligned}
\left(\nabla_{T} \cdot \nabla\right)^{\star} W_{2} & =\phi P W_{1} \\
W_{2} & =0 \text { near }|x|=0 .
\end{aligned}
$$


Hence

$$
\int_{t=\tau(x)} Q_{2} \phi \frac{1}{\rho} b_{l_{1}} \phi_{1} \frac{\partial}{\partial n} w d s=\int_{t=\tau(x)} \nabla \tau \cdot \nabla b_{l_{1}-1} e^{q} P W_{2} d s
$$

We can continue this procedure. Indeed, it follows, after $\left[l_{1} / 2\right]$ steps where $\left[l_{1} / 2\right]=l_{1} / 2$ if $l_{1}$ is even and is $\left(l_{1}-1\right) / 2$ if $l_{1}$ is odd, that

$$
\int_{t=\tau(x)} Q_{2} \phi \frac{1}{\rho} b_{l_{1}} \phi_{1} \frac{\partial}{\partial n} w d s=\int_{t=\tau(x)} \nabla \tau \cdot \nabla b_{\left[l_{1} / 2\right]} e^{q} P W_{\left[l_{1} / 2\right]+1} d s
$$

where $W_{i}$ solves

$$
\begin{aligned}
(\nabla \tau \cdot \Gamma)^{*} W_{i} & =\phi P W_{t-1} \\
W_{i} & =0 \text { near }|x|=0
\end{aligned}
$$

for $i=1, \cdots,\left[l_{1} / 2\right]+1$.

Therefore, by the Cauchy-Schwatz inequality, Proposition 2.1, and Rauch's Lemma

$$
\left|\int_{t=\tau(x)} Q_{2} \phi \frac{1}{\rho} b_{l_{1}} \phi_{1} \frac{\partial}{\partial n} w d s\right| \leq C \mid\left\|\psi b_{\left[l_{1} / 2\right]} \epsilon^{q}\right\|\left\|_{l_{1}-2\left[l_{1} / 2\right]}\right\| P W_{\left[l_{1} / 2\right]+1} \|_{2\left[l_{1} / 2\right]-l_{1}}
$$

with $C$ depending on $\left\|\sigma_{0}\right\|_{s}$, for some $s>n / 2$. Since $b_{i} e^{q}$ solves the $i$-th transport equation, Lemma 3.2 then implies that

$$
\left\|l^{\prime} b_{\left[l_{1} / 2\right]} C^{q}\right\|_{l_{1}-2\left[l_{1} / 2\right]} \leq C\left\|\phi \sigma_{0}\right\|_{l_{1}}
$$

Next, applying Lemma 3.2, after some simple calculations, we can obtain

$$
\left\|P W_{\left[l_{1} / 2\right]+1}\right\|_{2\left[l_{1} / 2\right]-l_{1}} \leq C\left\|\left.\phi e^{\sigma_{0}-q} Q_{2} \phi_{1}\left(\frac{\partial}{\partial n} w\right)\right|_{t=\tau(x)}\right\|_{l_{1}-1} .
$$

Now Lemma 3.4 and Lemma 3.5 may be applied to get

$$
\left\|P W_{\left[l_{1} / 2\right]+1}\right\|_{2\left[l_{1} / 2\right]-l_{1}} \leq C\|\Psi\|
$$

where the constant $C$ depends on $\left\|\sigma_{0}\right\|_{s}$ with $s>l_{1}-1 / 2+n / 2$.

Once again, a variant of the proof of Proposition 3.6, with no additional technical diffculty, will lead to the following result.

Proposition 3.8 The estimate

$$
\left|\int_{t=r(x)} Q_{2} \phi \frac{1}{\rho} a_{l_{1}} \phi_{1} \frac{\partial}{\partial n} w d s\right| \leq C\|\delta \sigma\|_{l_{1}}\|\Psi\|
$$

holds with the constant $C$ depending on $\left\|\sigma_{0}\right\|_{s}$, for $s>l_{1}+(n-1) / 2$. 


\section{References}

[1] G. Bao, Microlocal regularity of an inverse problem for the multidimensional wave equation, Ph.D. thesis, Dept. of Math. Sci., Rice Univ., Houston, Texas (1990).

[2] G. Bao and W. Symes, A trace theorem for solutions of linear partial differential equations, Math. Meth. in the Appl. Sci., Vol. 14, 5.53-562 (1991).

[3] G. Bao and W. Symes, Trace regularity result for a second order hyperbolic equation with nonsmooth coefficients, J. Math. Anal. \& Appl., to appear.

[4] M. Beals, Propagation of smoothness for nonlinear second order strictly hyperbolic equations, Proc. of Symp. in Pure Math. 43 (1985), 21-44.

[5] M. Beals, Propagation and Interaction of Singularities in Nonlinear Hyperbolic Problems, Birkhäuser, Boston, 1989.

[6] M. Beals and M. Reed, Propagation of singularities for hyperbolic pseudodifferential operators and applications to nonlinear problems, Comm. Pure Appl. Math. 35 (1982), $169-184$.

[7] G. Beylkin, Imaging of discontinuities in the inverse scattering problem by inversion of a. causal generalized Radon transform, J. Math. Phys. 26 (1985), 99-108.

[8] J. - M. Bony, Calcul symbolique et propagation des singularitéaires pour les équations aux dérivées partielles non-linéajres, Ann. Scien. Ecole. Norm. Sup. 14 (1981), 209-246.

[9] J. Chazarain and A. Piriou. Introduction to the Theory of Linear Partial Differential Equation, North Holland, Amsterdam, New York, 1982.

[10] R. Courant and D. Hilbert, Methods of Mathematical Physics, Vol.2, Wiley-Interscience, New York, 1962.

[11] J. J. Duistermaat, Fourier Integral Operators, Courant Institute Lecture Notes, New York, NY, 1973.

[12] F. G. Friedlander, Sound Pulses, Cambridge University Press, Cambridge, 1958.

[13] L. Hörmander, Fourier integral operators I, Acta Mathematica 127 (1971), 79-183.

[14] L. Hörmander, The Analysis of Linear Partial Differential Operators, Vol I, SpringerVerlag, New York, 1983.

[15] L. V. Kantorovich and G. P. Akilov, Functional Analysis in Normed Spaces, Russian ed., Moscow, 1959. Tr. by D. E. Brown, Pergamon, New York, 1964.

[16] Y. Meyer, Régularité des solutions des équations aux derivées partielles nonlinéaires, Sem. Bourbaki, No. 560 (1979-1980). 
[17] L. Nirenberg, Lectures on Linear Partial Differential Equations, CBMS Regional Conf. Ser. in Math., No. 17. Amer. Math. Soc., Providence, R.I., 1973.

[18] Rakesh, A linearized inverse problem for the wave equation, Comm. in P.D.E., 13 (1988), 573-601.

[19] J. Rauch, Singularities of solutions to semilinear wave equations, J. Math. Pures Appl. 58 (1979), 299-308.

[20] V. G. Romanov, Inverse Problems of Mathematical Physics, VNU Science Press BV, Great Britain, 1987.

[21] P. Sacks and W. Symes, Uniqueness and continuous dependence for a multidimensional hyperbolic inverse problem, Comm. in P.D.E., 10 (1985), 635-676.

[22] Z. Sun, Uniqueness theorems for inverse problems in wave propagation and electrostatics, Ph.D. Thesis, Dept. of Math., Univ. of California, Los Angeles, 1987.

[23] W. Symes, A trace theorem for solutions of the wave equation, and the remote determination of acoustic sources, Math. Meth. in the Appl. Sci. 5 (1983), 131-152.

[24] W. Symes, Some aspects of inverse problems in several dimensional wave propagation, Proc. Conference on Inverse Problems, SIAM-AMS Proceedings 14, ed. D. W. McLaughlin, Amer. Math. Soc., Providence, R.I., 1983.

[25] W. Symes, On the relation between coefficient and boundary values for solutions of Webster's horn equation, SIAM J. Math. Anal. 17 (1986), 1400-1420.

[26] M. Taylor, Pseudo-Differential Operators, Princeton Univ. Press, Princeton, N.J., 1981. 